\title{
MÉTODO DE DIFERENCIAS FINITAS PARA UN PROBLEMA DE VALOR DE FRONTERA UNIDIMENSIONAL
}

\section{THE FINITE- DIFERENCE METHOD FOR A ONE-DIMENSIONAL BOUNDARY-VALUE PROBLEM}

\author{
Luis Jaime Collantes Santisteban ${ }^{\mathrm{a}, \mathrm{b}}$, Samuel Collantes Santisteban ${ }^{\mathrm{a}}$
}

\section{RESUMEN}

En este trabajo se considera el problema de valor de frontera unidimensional dado en (1). Se aproxima la solución del problema mediante el método de diferencias finitas suponiendo que la función $c(x)$ es no negativa sobre $\, 1_{\_}^{-}$, lo que permite establecer la convergencia del método de aproximación. El uso del método de diferencias finitas, a la vez, involucra la solución de sistemas de ecuaciones lineales con matrices muy ralas, cuyos ceros están posicionados de una manera remarcable. Dichas matrices son de tipo tridiagonal. Para la solución de dichos sistemas se ha utilizado el método de Thomas.

Palabras clave: problema de valor de frontera unidimensional, diferencias finitas, matriz tridiagonal, método de Thomas, momento flexionante.

\section{ABSTRACT}

In this work the one-dimensional boundary-value problem given in (1) is considered. The solution of the problem by means of finite-difference method comes near supposing that the function $c(x)$ is nonnegative on $\mathbf{\prod}, 1_{-}^{-}$, which allows to establish the convergence of the considered method of approximation. The use of the finite-difference method, in turn, involves the solution of linear systems with very 'sparse' matrices, whose zeros are arranged in quite remarkable fashion. These matrices are of tridiagonal type. For the solution of these systems the Thomas' method has been used.

Key words: one-dimensional boundary-value problem, finite-difference, tridiagonal matrix, Thomas' method, bending moment.

\section{INTRODUCCIÓN}

Se denota a $C^{m}$ como el espacio vectorial de funciones reales $f: I \rightarrow R$ que son $m$ veces continuamente diferenciables, siendo $\quad m \in Z_{0}^{+}$. Además $f^{\prime}, f^{\prime \prime} \mathrm{y} f^{(n)} ; n \geq 3$ denotan las derivadas sucesivas de " $f$ ".

Se considera el siguiente problema de valor de frontera (PVF): Dados $c, f \in C^{0} \mathbf{Q}, 1^{-}$y $\alpha, \beta$ constantes, hallar $\left.u \in C^{2}, 1\right]$ satisfaciendo:

$$
\left\{\begin{array}{c}
-u^{\prime \prime}(x)+c(x) u(x)=f(x), \quad 0<x<1, \\
u(0)=\alpha, \quad u(1)=\beta .
\end{array}\right.
$$

Resolver este problema por métodos exactos generalmente es posible cuando la función $c(x)$ es constante. Para otros casos es imprescindible utilizar métodos numéricos ([1], [2]).

Este problema es importante porque representa diversas aplicaciones de gran relevancia en Ingeniería ([3], [4]).

En [3] encontramos un problema de origen físico de este tipo, el cual corresponde a la flexión de una viga de una unidad de longitud, estirada a lo largo de sus semiejes por una fuerza " $P$ ", sujeta a una carga transversal $f(x) d x$ por el elemento $d x$ y simplemente soportada en sus extremos " 0 " $y$ " 1 ".

El momento flexionante $u(x)$ en la abscisa $x$, resulta ser la solución de un problema de valor de frontera del tipo dado con

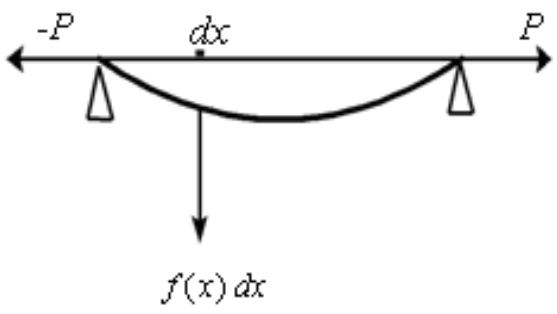

Fig. 1. Flexión de una viga

$c(x)=P / E I(x)$ - donde $E$ es el módulo de Young de elasticidad del material de la viga e $I(x)$ representa el momento de inercia de la sección transversal de la viga con respecto al eje $x$, con $\alpha=\beta=0$.

${ }^{a}$ Facultad de Ingeniería, Universidad Católica Santo Toribio de Mogrovejo, Perú

${ }^{\mathrm{b}}$ Departamento de Matemáticas, Universidad Nacional Pedro Ruiz Gallo, Perú 
Al suponer que la función " $c$ " es no negativa sobre el intervalo $\left[, 1^{-}\right.$, se demuestra en [5] que el problema tiene única solución, denotada por " $\varphi$ ".

Surge la necesidad de hallar una vía para aproximar los valores de la solución. Uno de los métodos para lograr esto es el método de diferencias finitas, el cual es un camino de obtención de una aproximación de la solución " $\varphi$ " en los nodos de la malla, es decir se busca un vector $u_{h}=\left(\begin{array}{c}u_{1} \\ u_{2} \\ \vdots \\ u_{N}\end{array}\right) \in R^{N}$, tal que los $u_{i}$ estén "cerca" de $\varphi \varangle_{i}^{-}$, para $i=1, \ldots, N$.

\section{MÉTODO DE DIFERENCIAS FINITAS}

Se ha utilizado el método numérico de diferencias finitas para plantear el problema (1) en forma alternativa como un sistema tridiagonal de ecuaciones lineales, cuya solución ha sido posible aplicando el algoritmo de Thomas [6], implementado computacionalmente.

En el método de diferencias finitas se pueden distinguir los siguientes pasos:

\section{Discretización del dominio}

El intervalo $\$, 1^{-}$es dividido en un número finito de subintervalos igualmente espaciados de longitud " $h$ ", llamado diferencia finita $h>0$.

Definición 1. Una malla en el dominio $I=\boldsymbol{\downarrow}, 1_{\text {_}}$, es un conjunto de puntos $\tau=\left\{I\right.$, tal que cada $x_{i}$ satisface $x_{i+1}=x_{i}+h, \quad i=0, \ldots, N$.

Estos puntos también son llamados nodos y generalmente se eligen de la forma

$$
x_{i}=i h, \quad i=0,1,2, \ldots, N
$$

donde $N+1$ es el número de subdominios.

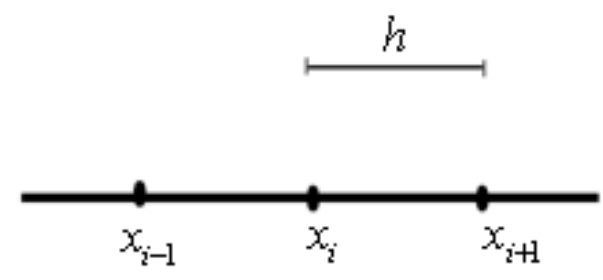

Fig. 2. Malla unidimensional

\section{Discretización de la función}

Definición 2. Una función discreta $v$, es aquella que está definida en una malla $\tau$, tal que a cada punto $x_{i}$ le asocia un número $v_{i}$.
Definición 3. Sea $f=f_{\text {, }}$, una función discreta definida sobre una malla $\tau$, su norma euclideana se define como

$$
\|f\|=(f, f\rangle_{h} \boldsymbol{T}^{2}=\left(\sum_{i=1}^{N} h f_{i}^{2}\right)^{1 / 2},
$$

donde $h$ es generalmente asociado al tamaño de la malla $h=\max _{0 \leq i \leq N}-x_{i}$ :

También existen otras normas para la función discreta, tal como la norma del máximo

$$
\|f\|_{\infty}=\left.\max _{1 \leq i \leq N} h f_{i}\right|^{-}
$$

\section{Discretización de la ecuación diferencial}

En el problema (1), se supone que la solución $\left.\varphi \in C^{4}, 1\right]$ y que $x_{i}=i h$; usando la fórmula de Taylor para $i=1, \ldots, N$, se tiene que:

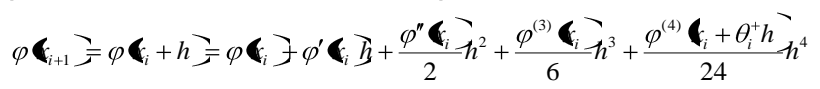

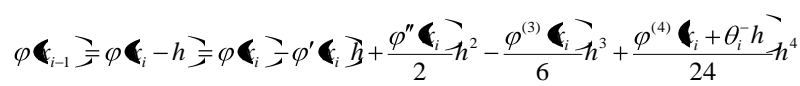

con $-1<\theta_{i}^{-}<0<\theta_{i}^{+}<1$. Al sumar (2) y (3) se tiene:

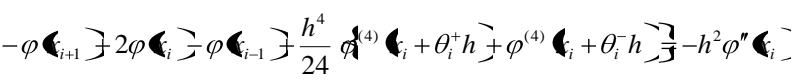

Por el "teorema del valor intermedio" [7], para cada $1 \leq i \leq N: \quad \exists x_{i}+\theta_{i} h_{-}^{-} \in\left\langle x_{i}+\theta_{i}^{-} h, x_{i}+\theta_{i}^{+} h\right\rangle$, con

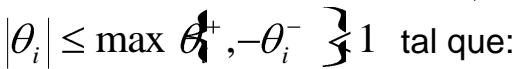

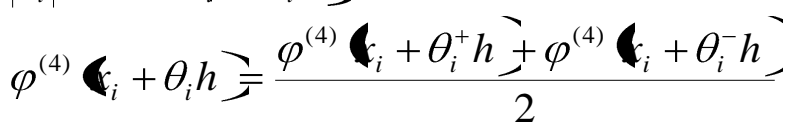

reemplazando (5) en (4):

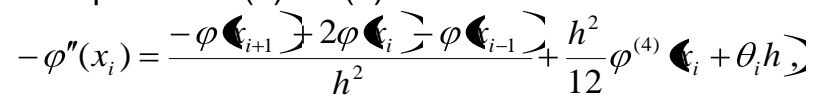

al sustituir (6) en (1) se obtiene:

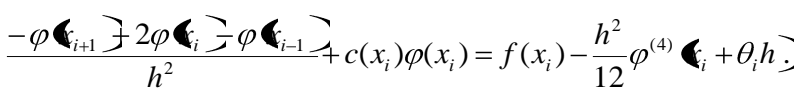

Considerando en (7) la siguiente notación:

$\varphi_{i}=\varphi\left(x_{i}\right), c_{i}=c\left(x_{i}\right), f_{i}=f\left(x_{i}\right), 1 \leq i \leq N$, se tiene: para $i=1$ :

$$
\frac{-\varphi_{2}+2 \varphi_{1}-\alpha}{h^{2}}+c_{1} \varphi_{1}=f_{1}-\frac{h^{2}}{12} \varphi^{(4)}\left(x_{1}+\theta_{1} h\right),
$$

para $2 \leq i \leq N-1$ :

$$
\frac{-\varphi_{i+1}+2 \varphi_{i}-\varphi_{i-1}}{h^{2}}+c_{i} \varphi_{i}=f_{i}-\frac{h^{2}}{12} \varphi^{(4)}\left(x_{i}+\theta_{i} h\right),
$$

para $i=N$ :

$\frac{-\beta+2 \varphi_{N}-\varphi_{N-1}}{h^{2}}+c_{N} \varphi_{N}=f_{N}-\frac{h^{2}}{12} \varphi^{(4)}\left(x_{N}+\theta_{N} h\right)$,

ordenando: 


$$
\begin{aligned}
-\frac{\alpha}{h^{2}}+\frac{2 \varphi_{1}-\varphi_{2}+c_{1} h^{2} \varphi_{1}}{h^{2}}=f_{1}-\frac{h^{2}}{12} \varphi^{(4)}\left(x_{1}+\theta_{1} h\right) \\
\frac{-\varphi_{1}+2 \varphi_{2}-\varphi_{3}+c_{2} h^{2} \varphi_{2}}{h^{2}}=f_{2}-\frac{h^{2}}{12} \varphi^{(4)}\left(x_{2}+\theta_{2} h\right) \\
\vdots \\
\frac{-\varphi_{N-2}+2 \varphi_{N-1}-\varphi_{N}+c_{N-1} h^{2} \varphi_{N-1}}{h^{2}}=f_{N-1}-\frac{h^{2}}{12} \varphi^{(4)}\left(x_{N-1}+\theta_{N-1} h\right) \\
-\frac{\beta}{h^{2}}+\frac{-\varphi_{N-1}+2 \varphi_{N}+c_{N} h^{2} \varphi_{N}}{h^{2}}=f_{N}-\frac{h^{2}}{12} \varphi^{(4)}\left(x_{N}+\theta_{N} h\right)
\end{aligned}
$$

Este sistema de " $N$ " ecuaciones puede ser escrito matricialmente como:

donde:

$$
\mathbf{A}_{h} \varphi_{h}=\mathbf{b}_{h}+\boldsymbol{\varepsilon}_{h}(\varphi)
$$

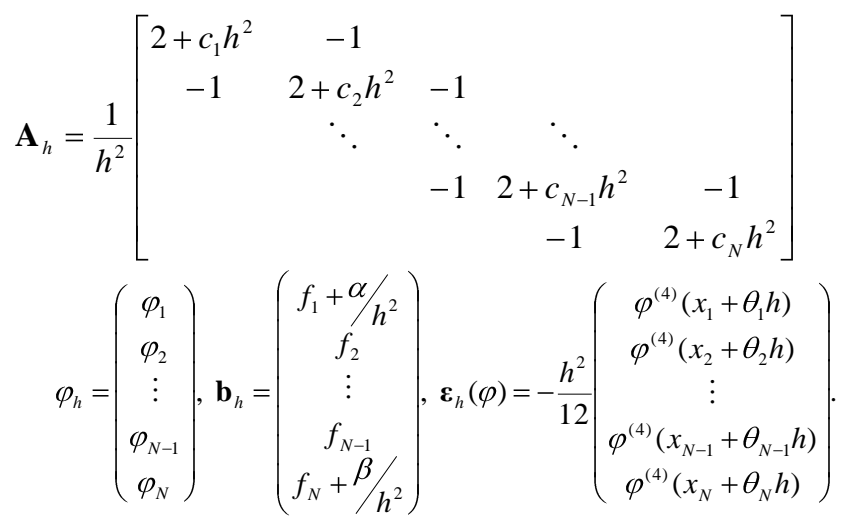

El vector " $\boldsymbol{\varepsilon}_{h}(\varphi)$ " tiende a cero a medida que la malla de medida " $h$ " mejora por el factor " $h^{2}$ ". Se define el problema asociado: hallar $u_{h} \in R^{N}$, solución de la ecuación matricial $\quad \mathbf{A}_{h} \mathbf{u}_{h}=\mathbf{b}_{h}$ (8)

En [5] se demuestra el siguiente teorema.

Teorema 1. Si la solución $\varphi \in C^{4} \Phi, 1_{-}^{-}$y se tiene que la función " $c$ " es no negativa, entonces

$\max _{1 \leq i \leq N}\left|u_{i}-\varphi\left(x_{i}\right)\right|=\left\|\mathbf{u}_{h}-\varphi_{h}\right\|_{\infty} \leq\left\{\frac{1}{96} \sup _{0 \leq x \leq 1}\left|\varphi^{4}(x)\right|\right\} h^{2}$

\section{RESULTADOS Y DISCUSIÓN}

La primera prueba numérica correspondió al PVF:

$\left\{\begin{array}{c}-u^{\prime \prime}(x)+x u(x)=\left(1+2 x-x^{2}\right) \exp (x), \quad 0<x<1, \\ u(0)=1, \quad u(1)=0 .\end{array}\right.$

con solución exacta $\varphi(x)=(1-x) \exp (x)$.

Tabla 1: Resultados de la primera prueba numérica

\begin{tabular}{ccc}
\hline \hline$N$ & $h=1 / N+1$ & $\left\|u_{h}-\varphi_{h}\right\|_{\infty}$ \\
\hline 1 & $1 / 2$ & 0.0143297858 \\
3 & $1 / 4$ & 0.0036570354 \\
7 & $1 / 8$ & 0.0009192189 \\
15 & $1 / 16$ & 0.0002324750 \\
31 & $1 / 32$ & 0.0000581416 \\
63 & $1 / 64$ & 0.0000145448 \\
127 & $1 / 128$ & 0.0000036346
\end{tabular}

\begin{tabular}{lll}
255 & $1 / 256$ & 0.0000009278 \\
511 & $1 / 512$ & 0.0000001734 \\
\hline
\end{tabular}

La segunda prueba numérica consideró el PVF:

$\left\{\begin{array}{c}-u^{\prime \prime}(x)+(x+1) u(x)=-x\left(6-x^{2}-x^{3}\right), \quad 0<x<1, \\ u(0)=0, \quad u(1)=1 .\end{array}\right.$

con solución exacta $\varphi(x)=x^{3}$.

Tabla 2: Resultados de la segunda prueba numérica

\begin{tabular}{ccc}
\hline \hline$N$ & $h=1 / N+1$ & $\left\|u_{h}-\varphi_{h}\right\|_{\infty}$ \\
\hline 1 & $1 / 2$ & 0.4210526316 \\
3 & $1 / 4$ & 0.0000000000 \\
7 & $1 / 8$ & 0.0000000001 \\
15 & $1 / 16$ & 0.0000000006 \\
31 & $1 / 32$ & 0.0000000026 \\
63 & $1 / 64$ & 0.0000000016 \\
127 & $1 / 128$ & 0.0000000103 \\
255 & $1 / 256$ & 0.0000000065 \\
511 & $1 / 512$ & 0.0000000760 \\
\hline \hline
\end{tabular}

La tercera prueba numérica correspondió al PVF:

$$
\left\{\begin{array}{c}
-u^{\prime \prime}(x)+2 \pi^{2} u(x)=-\frac{9 \pi^{2}}{40}\left[\operatorname{sen}\left(\frac{\pi x}{2}\right)+3 \cos \left(\frac{\pi x}{2}\right)\right], \quad 0<x<1, \\
u(0)=-0.3, \quad u(1)=-0.1 .
\end{array}\right.
$$

con solución exacta

$$
\varphi(x)=-\frac{1}{10}\left[\operatorname{sen}\left(\frac{\pi \mathrm{x}}{2}\right)+3 \cos \left(\frac{\pi \mathrm{x}}{2}\right)\right] .
$$

Tabla 3: Resultados de la tercera prueba numérica

\begin{tabular}{ccc}
\hline \hline$N$ & $h=1 / N+1$ & $\left\|u_{h}-\varphi_{h}\right\|_{\infty}$ \\
\hline 1 & $1 / 2$ & 0.0131530529 \\
3 & $1 / 4$ & 0.0003350832 \\
7 & $1 / 8$ & 0.0000860024 \\
15 & $1 / 16$ & 0.0000217979 \\
31 & $1 / 32$ & 0.0000054537 \\
63 & $1 / 64$ & 0.0000013628 \\
127 & $1 / 128$ & 0.0000003313 \\
255 & $1 / 256$ & 0.0000001721 \\
511 & $1 / 512$ & 0.0000001653 \\
\hline \hline
\end{tabular}

Para cada PVF se obtuvo el respectivo sistema tridiagonal de ecuaciones lineales del tipo (8); la implementación computacional del algoritmo de Thomas condujo a los resultados numéricos mostrados en las tablas dadas. El valor de $N$ en cada paso se ha ido tomando de tal manera que la medida de la malla $h$ se reduce a la mitad de la medida del paso previo. Los resultados confirman que la solución aproximada efectivamente converge a la solución exacta.

\section{CONCLUSIONES}

Muchos problemas, particularmente provenientes de la Física, conducen a la solución del primer problema fundamental del análisis numérico de matrices: resolver un sistema de ecuaciones lineales. En este caso, el método de 
diferencias finitas aplicado al problema de valor de frontera (1) conduce a resolver un sistema tridiagonal de ecuaciones lineales. La suposición de que la función $c(x)$ es no negativa permite afirmar, a través del teorema 1, que la solución aproximada converge a la solución exacta en un orden de $h^{2}$.

El método de diferencias finitas ha permitido aproximar la solución de la ecuación diferencial en los nodos de una malla. El vector error " $\boldsymbol{\varepsilon}_{h}(\varphi)$ " se hace más pequeño a medida que $h$ tiende a cero.

La programación computacional del algoritmo de Thomas ha permitido obtener los resultados numéricos que ayudan a comprobar experimentalmente que la solución aproximada converge hacia la solución exacta.

\section{REFERENCIAS BIBLIOGRÁFICAS}

[1]. Chapra S., Canale R. Métodos Numéricos para Ingenieros. Mc Graw Hill, México, 2007.

[2]. García A., et al. Ecuaciones diferenciales ordinarias. Teoría y problemas. Métodos exactos. Métodos Numéricos. Estudio cualitativo. CLAGSA, España, 2006.

[3]. Pytel A., Singer F. Resistencia de Materiales. Harla, México, 1994.

[4]. Zill D., Cullen M., Ecuaciones diferenciales con problemas de valor en la frontera. Thomson, México, 2006.

[5]. Ciarlet Ph. Introduction to numerical linear algebra and optimisation. Cambridge University Press, Great Britain, 1989. pp. 71-72.

[6]. Nakamura S. Métodos Numéricos aplicados con software. Prentice Hall, México, 1992. p. 358.

[7]. Alder H., Figueroa E. Introducción al Análisis Numérico. Universidad de Concepción, Chile, 1994.

E-mail: Icollantes@usat.edu.pe 